\title{
Comment on "Indirect $\mathrm{CP}$ violation in the B-system"
}

\author{
L. Lavoura \\ Universidade Técnica de Lisboa \\ Centro de Física das Interacções Fundamentais \\ Instituto Superior Técnico, P-1096 Lisboa Codex, Portugal \\ Email i009@beta.ist.utl.pt. Telephone (+351 1) 8419093. Fax (+351 1) 8419143.
}

24 June 1998

\begin{abstract}
In a recent paper, Bañuls and Bernabéu have claimed the existence of a new form of indirect $\mathrm{CP}$ violation which would have its most prominent manifestation in the $B_{d}-\bar{B}_{d}$ system. I analyse this claim in detail. I emphasize the fact that it is necessary to take into account the precise experimental method if one is to identify correctly the weak phase that one is about to measure.
\end{abstract}

\section{Introduction}

In a recent paper [1], Bañuls and Bernabéu (BB) have claimed that:

1. It is possible to define a complex rephasing-invariant parameter $\epsilon$ in the mixing of each neutral-meson system (for instance, the $K-\bar{K}$ or the $B_{d^{-}}$ $\bar{B}_{d}$ system), such that $\operatorname{Re} \epsilon$ and $\operatorname{Im} \epsilon$ are two independent, measurable $\mathrm{CP}$-violating quantities.

2. While

$$
\frac{2 \operatorname{Re} \epsilon}{1+|\epsilon|^{2}}=\frac{|p|^{2}-|q|^{2}}{|p|^{2}+|q|^{2}}
$$

is the usual parameter of indirect $\mathrm{CP}$ violation, which is experimentally known to be no larger than $10^{-1}$ in the $B_{d^{-}} \bar{B}_{d}$ system [2], $\operatorname{Im} \epsilon$ corresponds to a new form of indirect $\mathrm{CP}$ violation, which should be particularly large 
in that system. BB have predicted that

$$
-0.74<\frac{2 \operatorname{Im} \epsilon}{1+|\epsilon|^{2}}<-0.36
$$

in the $B_{d}-\bar{B}_{d}$ system.

3. By observing the time dependence of the flavour-specific decays of states which at initial time were tagged to be one of the $\mathrm{CP}$ eigenstates of the neutral-meson system, one may construct an asymmetry which allows one to determine both the real and the imaginary parts of $\epsilon$.

The results advertised by $\mathrm{BB}$ are surprising for many reasons. BB not only claim the existence of an hitherto unnoticed, completely new form of $\mathrm{CP}$ violation in the mixing of neutral mesons, they also suggest an experiment to measure it, and they are even able to predict its standard-model value. The prediction is baffling in that it is based on nothing more than the well-known standard-model box diagram for $M_{12}$ in the $B_{d}-\bar{B}_{d}$ system.

These amazing results have entailed me to analyse BB's work in detail. I found that BB's interpretation of some calculations is mostly wrong.

In particular, the prediction in eqn (2) basically follows from the identification

$$
\frac{2 \operatorname{Im} \epsilon}{1+|\epsilon|^{2}}=-\frac{\operatorname{Im}\left[\left(V_{t b} V_{t d}^{*}\right)^{2}\right]}{\left|V_{t b} V_{t d}\right|^{2}},
$$

where $V$ is the Cabibbo-Kobayashi-Maskawa mixing matrix. Equation (3) arouses suspicion, because the combination $\left(V_{t b} V_{t d}^{*}\right)^{2}$ is not invariant under a rephasing of the $d$ - and $b$-quark fields, and therefore the imaginary part of that combination is not measurable. Indeed, I find that eqn (3) neglects the CPtransformation phases of the quarks, a procedure which, although commonplace in the literature, is illegitimate.

Moreover, BB have explicitly claimed that the product

$$
\left\langle f|T| B_{+}\right\rangle^{*}\left\langle f|T| B_{-}\right\rangle
$$

- where $B_{+}$and $B_{-}$are the CP eigenstates of the $B_{d}-\bar{B}_{d}$ system - is not phaseconvention-dependent; however, it is clear that independent rephasings of $\left|B_{+}\right\rangle$ and of $\left|B_{-}\right\rangle$do change the phase of that product. This fact suggests that, when $\mathrm{BB}$ write that $\epsilon$ is rephasing-invariant, they are not taking into account the freedom that one has to independently rephase all the kets. This suspicion proves to be true.

\section{The CP-violating asymmetry}

It is convenient to start by analysing BB's proposal for the measurement of a well-defined time-dependent $\mathrm{CP}$-violating asymmetry. The proposed experi- 
ment basically consists in the following. 1 One uses a state which, at initial time $t=0$, is a coherent superposition of $\left|B_{d}\right\rangle$ and $\left|\bar{B}_{d}\right\rangle$ in which there is an equal probability of finding $B_{d}$ and of finding $\bar{B}_{d}$. One may write such a state, in all generality, as

$$
\left|B_{+}\right\rangle=\frac{e^{i \alpha_{+}}}{\sqrt{2}}\left(\left|B_{d}\right\rangle+e^{i \zeta}\left|\bar{B}_{d}\right\rangle\right) .
$$

This state evolves into $\left|B_{+}(t)\right\rangle$ at proper time $t>0$. One measures the probabilities of finding $B_{d}$ and of finding $\bar{B}_{d}$ in $B_{+}(t)$ and one constructs the corresponding asymmetry:

$$
A_{+}^{\mathrm{CP}}(t) \equiv \frac{\left|\left\langle B_{d} \mid B_{+}(t)\right\rangle\right|^{2}-\left|\left\langle\bar{B}_{d} \mid B_{+}(t)\right\rangle\right|^{2}}{\left|\left\langle B_{d} \mid B_{+}(t)\right\rangle\right|^{2}+\left|\left\langle\bar{B}_{d} \mid B_{+}(t)\right\rangle\right|^{2}} .
$$

Obviously, $A_{+}^{\mathrm{CP}}(t) \neq 0$ represents a violation of $\mathrm{CP}$ : if an initial state in which there is an equal probability of finding a particle and its antiparticle evolves into a final state in which the probabilities of finding the particle and the antiparticle are different, then $\mathrm{CP}$ is violated. The asymmetry $A_{+}^{\mathrm{CP}}(t)$ (or its time-integrated version, which of course contains less information) is the observable whose measurement has been proposed by BB.

In order to compute $A_{+}^{\mathrm{CP}}(t)$ it is necessary to use the eigenstates of mass, which I write as

$$
\begin{aligned}
& \left|B_{1}\right\rangle=p_{1}\left|B_{d}\right\rangle+q_{1}\left|\bar{B}_{d}\right\rangle \\
& \left|B_{2}\right\rangle=p_{2}\left|B_{d}\right\rangle-q_{2}\left|\bar{B}_{d}\right\rangle .
\end{aligned}
$$

I assume these states to be normalized: $\left|p_{1}\right|^{2}+\left|q_{1}\right|^{2}=\left|p_{2}\right|^{2}+\left|q_{2}\right|^{2}=1$. It follows from CPT invariance in the mixing that

$$
\frac{q_{1}}{p_{1}}=\frac{q_{2}}{p_{2}} \equiv \frac{q}{p}=\sqrt{\frac{1-\delta}{1+\delta}} e^{i \chi},
$$

where

$$
\delta=\frac{2 \operatorname{Re} \epsilon}{1+|\epsilon|^{2}}
$$

- see eqn (11) - is the usual T- and CP-violating quantity. Usually one makes a convention for the relative phase of $\left|B_{1}\right\rangle$ and $\left|B_{2}\right\rangle$ such that $p_{1}=p_{2} \equiv p$ and $q_{1}=q_{2} \equiv q$.

The phase

$$
\theta \equiv \zeta-\arg \frac{q}{p}
$$

\footnotetext{
${ }^{1}$ For definiteness I always work in terms of the $B_{d}-\bar{B}_{d}$ system, which is the one for which BB's "discoveries" are supposed to be most important.

${ }^{2}$ The probabilities in eqn (5) may be measured by observing the decays into flavour-specific modes, like for instance the semileptonic modes $l^{ \pm} X^{\mp}$, of $B_{+}(t)$. When doing this one must assume that $\left|\left\langle l^{+} X^{-}|T| B_{d}\right\rangle\right|=\left|\left\langle l^{-} X^{+}|T| \bar{B}_{d}\right\rangle\right| ;$ this equality follows from CPT invariance.
} 
is invariant under a change of the relative phase of $\left|B_{d}\right\rangle$ and $\left|\bar{B}_{d}\right\rangle$, contrary to what happens with the phases $\zeta$ and $\arg q / p$ separately - $c f$. eqns (5) and (7).

The states $\left|B_{k}\right\rangle(k=1,2)$ have exponential evolution laws: $\left|B_{k}(t)\right\rangle=$ $\exp \left(-i \lambda_{k} t\right)\left|B_{k}\right\rangle$ with $\lambda_{k}=m_{k}-(i / 2) \gamma_{k}$. Defining $\Delta m \equiv m_{2}-m_{1}$ and $\Delta \Gamma \equiv \gamma_{2}-\gamma_{1}$, one finds

$$
\begin{aligned}
A_{+}^{\mathrm{CP}}(t)= & {\left[\delta \cosh (\Delta \Gamma t / 2)+\delta \sqrt{1-\delta^{2}} \cos \theta \sinh (\Delta \Gamma t / 2)\right.} \\
& \left.-\delta \cos (\Delta m t)-\sqrt{1-\delta^{2}} \sin \theta \sin (\Delta m t)\right] \\
& \times\left[\cosh (\Delta \Gamma t / 2)+\sqrt{1-\delta^{2}} \cos \theta \sinh (\Delta \Gamma t / 2)\right. \\
& \left.-\delta^{2} \cos (\Delta m t)-\delta \sqrt{1-\delta^{2}} \sin \theta \sin (\Delta m t)\right]^{-1} .
\end{aligned}
$$

By measuring $A_{+}^{\mathrm{CP}}(t)$ one may in principle find $\delta$ and $\theta$. It is clear from eqn (11) that $\sin \theta \neq 0$ represents $\mathrm{CP}$ violation, just as $\delta \neq 0$. Now, we know that $\delta$ is small, and therefore we may write

$$
A_{+}^{\mathrm{CP}}(t) \approx \frac{\delta[\cosh (\Delta \Gamma t / 2)+\cos \theta \sinh (\Delta \Gamma t / 2)-\cos (\Delta m t)]-\sin \theta \sin (\Delta m t)}{\cosh (\Delta \Gamma t / 2)+\cos \theta \sinh (\Delta \Gamma t / 2)-\delta \sin \theta \sin (\Delta m t)} .
$$

Notice however that it is illegitimate to assume $\sin \theta$ to be small; in general, $\theta$ depends on $\zeta$, i.e., it depends on the initial state $\left|B_{+}\right\rangle$that one uses. Therefore, $\theta$ may be large or small depending on the particular $B_{+}$. It is unwarranted to assume $\theta$ to be of the same order of magnitude as $\delta$, like BB have done.

\section{Definition of $\epsilon$}

One may unify the two real CP-violating quantities $\delta$ and $\sin \theta$ in a single complex CP-violating parameter $\epsilon$ by means of a simple - albeit meaningless - trick. Let us consider another coherent superposition of $\left|B_{d}\right\rangle$ and $\left|\bar{B}_{d}\right\rangle$ in which the probabilities of finding $B_{d}$ and of finding $\bar{B}_{d}$ are equal and which is orthogonal to $\left|B_{+}\right\rangle$:

$$
\left|B_{-}\right\rangle=\frac{e^{i \alpha_{-}}}{\sqrt{2}}\left(\left|B_{d}\right\rangle-e^{i \zeta}\left|\bar{B}_{d}\right\rangle\right) .
$$

We use, together with $\mathrm{BB}, p_{1}=p_{2} \equiv p$ and $q_{1}=q_{2} \equiv q$. Now, if one writes

$$
\begin{aligned}
& \left|B_{1}\right\rangle=\frac{1}{\sqrt{1+|\epsilon|^{2}}}\left(\left|B_{+}\right\rangle+\epsilon\left|B_{-}\right\rangle\right), \\
& \left|B_{2}\right\rangle=\frac{1}{\sqrt{1+|\epsilon|^{2}}}\left(\left|B_{-}\right\rangle+\epsilon\left|B_{+}\right\rangle\right),
\end{aligned}
$$

then this means that: 
1. One is assuming the phases $\alpha_{+}$and $\alpha_{-}$to be equal.

2. One is fixing

$$
\epsilon=\frac{\delta+i \sqrt{1-\delta^{2}} \sin \theta}{1+\sqrt{1-\delta^{2}} \cos \theta} .
$$

This is in fact what BB have implicitly done.

It follows from eqn (15) that

$$
\frac{2 \epsilon}{1+|\epsilon|^{2}}=\delta+i \sqrt{1-\delta^{2}} \sin \theta .
$$

Then, both $2 \operatorname{Re} \epsilon /\left(1+|\epsilon|^{2}\right)=\delta$ and $2 \operatorname{Im} \epsilon /\left(1+|\epsilon|^{2}\right) \approx \sin \theta$ are measurable $\mathrm{CP}$-violating parameters. In this sense, it is true that "both $\operatorname{Re} \epsilon$ and $\operatorname{Im} \epsilon$ are observable quantities", as BB have written; on the other hand, $\epsilon$ cannot really be considered a phase-convention-independent parameter, because its definition depends on the phase convention $\alpha_{+}=\alpha_{-}$. Also, $\epsilon$, besides being phaseconvention-dependent - it depends on the relative phase of $\left|B_{+}\right\rangle$and $\left|B_{-}\right\rangle-$, is a completely artificial parameter, for it joins together $\delta$, which only depends on the mixing of $B_{d}$ and $\bar{B}_{d}$, and $\theta$, which depends on the specific initial state $B_{+}$ used in a particular experiment.

Therefore, BB's assertion that $\operatorname{Im} \epsilon$ represents a new form of $\mathrm{CP}$ violation in the mixing of $B_{d}$ and $\bar{B}_{d}$ is wrong. BB have been taken to believe this because, instead of working with an arbitrary $B_{+}$- an arbitrary $\zeta$ - to begin with, they have assumed $B_{+}$to be an eigenstate of CP. Unfortunately, as we shall see in section 5 , that assumption cannot be realized in a concrete experiment.

\section{The phase of $q / p$}

Up to now, the phase $\zeta$ has not been specified and, as such, $\theta$ is free too. In their paper, BB have specifically suggested using as initial states eigenstates of CP. Thus, if

$$
\begin{aligned}
& \mathcal{C P}\left|B_{d}\right\rangle=e^{i \xi}\left|\bar{B}_{d}\right\rangle, \\
& \mathcal{C P}\left|\bar{B}_{d}\right\rangle=e^{-i \xi}\left|B_{d}\right\rangle,
\end{aligned}
$$

then $\mathrm{BB}$ would want to use $\zeta=\xi$. Clearly, $B_{+}$is then the CP-even eigenstate and $B_{-}$is the $\mathrm{CP}$-odd eigenstate.

For this very specific choice, BB have proceeded to compute $\theta$. In order to do this they have computed the phase of $q / p$. Assuming $\left|\Gamma_{12}\right| \ll\left|M_{12}\right|$, one has 3.

$$
\frac{q}{p}=\sqrt{\frac{M_{12}^{*}}{M_{12}}}
$$


The matrix element $M_{12}$ is given by the standard-model box diagram, which is dominated by intermediate top quarks. One obtains

$$
\frac{M_{12}^{*}}{M_{12}}=\frac{\left(V_{t b}^{*} V_{t d}\right)^{2}\left\langle\bar{B}_{d}\left|\left[\bar{b} \gamma^{\mu}\left(1-\gamma_{5}\right) d\right]\left[\bar{b} \gamma_{\mu}\left(1-\gamma_{5}\right) d\right]\right| B_{d}\right\rangle}{\left(V_{t b} V_{t d}^{*}\right)^{2}\left\langle B_{d}\left|\left[\bar{d} \gamma_{\mu}\left(1-\gamma_{5}\right) b\right]\left[\bar{d} \gamma^{\mu}\left(1-\gamma_{5}\right) b\right]\right| \bar{B}_{d}\right\rangle} .
$$

The matrix elements may be related to each other by means of the CP symmetry of the strong interactions. In order to do this one must use, besides eqns (17), the $\mathrm{CP}$ transformation of the quark fields, which reads

$$
\begin{aligned}
& (\mathcal{C P}) d(\mathcal{C P})^{\dagger}=e^{i \xi_{d}} \gamma^{0} C \bar{d}^{T}, \\
& (\mathcal{C P}) \bar{b}(\mathcal{C P})^{\dagger}=-e^{-i \xi_{b}} b^{T} C^{-1} \gamma^{0},
\end{aligned}
$$

where $\xi_{d}$ and $\xi_{b}$ are arbitrary CP-transformation phases. It follows that

$$
\frac{q}{p}= \pm \frac{V_{t b}^{*} V_{t d}}{V_{t b} V_{t d}^{*}} e^{i\left(\xi+\xi_{d}-\xi_{b}\right)}
$$

Therefore,

$$
\begin{aligned}
\theta & =\xi-\arg \frac{q}{p} \\
& =2 \arg \left(V_{t b} V_{t d}^{*}\right)+\xi_{b}-\xi_{d}(\bmod \pi) .
\end{aligned}
$$

This is exactly the path followed by $\mathrm{BB}$, with one important exception: $B B$ omitted the arbitrary phases $\xi_{b}$ and $\xi_{d}$ in the $\mathrm{CP}$ transformation of the quark fields, implicitly setting them to zero. They obtained $\theta=2 \arg \left(V_{t b} V_{t d}^{*}\right)+\pi$, which depends on the phases chosen for the $b$ - and $d$-quark fields. But, as $\theta$ is an observable phase, it must be rephasing-invariant. Thus, it is clear that BB's procedure is meaningless.

One is not allowed to light-heartedly discard the arbitrary phases $\xi_{b}$ and $\xi_{d}$. The CP-transformation phases of the quark fields are essential, as may be seen for instance when one sets out to study the CP-invariance conditions for the charged-current Lagrangian

$$
\frac{g}{2 \sqrt{2}} \sum_{\alpha=u, c, t} \sum_{k=d, s, b}\left[W_{\mu}^{+} V_{\alpha k} \bar{\alpha} \gamma^{\mu}\left(1-\gamma_{5}\right) k+W_{\mu}^{-} V_{\alpha k}^{*} \bar{k} \gamma^{\mu}\left(1-\gamma_{5}\right) \alpha\right] .
$$

The most general CP transformation is

$$
\begin{aligned}
(\mathcal{C P}) W_{\mu}^{+}(\mathcal{C P})^{\dagger} & =-e^{i \xi_{W}} W^{\mu-} \\
(\mathcal{C P}) \bar{\alpha}(\mathcal{C P})^{\dagger} & =-e^{-i \xi_{\alpha}} \alpha^{T} C^{-1} \gamma^{0}, \\
(\mathcal{C P}) k(\mathcal{C P})^{\dagger} & =e^{i \xi_{k}} \gamma^{0} C \bar{k}^{T}
\end{aligned}
$$

If there is to be $\mathrm{CP}$ invariance, the $\mathrm{CP}$-transformation phases must be chosen such that

$$
V_{\alpha k}=e^{i\left(-\xi_{W}+\xi_{\alpha}-\xi_{k}\right)} V_{\alpha k}^{*} .
$$


Clearly, if it was not for the freedom allowed by the phases $\xi_{\alpha}$ and $\xi_{k}$, it would suffice that any two elements of $V$ have different phases for CP to be violated. It is well known that things are not so: a whole quartet $V_{\alpha k} V_{\beta j} V_{\alpha j}^{*} V_{\beta k}^{*}$ must be non-real in order for there to be $\mathrm{CP}$ violation.

\section{A practical experiment}

From the previous section, and in particular from eqn 22 , one gathers that the phase $\xi-\arg q / p$ is not measurable. On the other hand, one knows from eqn (11) that the phase $\zeta-\arg q / p$ is measurable. One can only conclude that $\zeta$ can never be equal to $\xi$ in a real experiment.

The phase $\zeta$ in the initial state $B_{+}$must be such that:

1. It includes the CP-transformation phase $\xi+\xi_{d}-\xi_{b}$, which is going to cancel out a similar term in $\arg q / p$ - see eqn (21).

2. It includes the phase of some elements of $V$, in such a way that $\zeta-\arg q / p$ is invariant under a rephasing of the quark fields.

Taking $\zeta=\xi$ does not satisfy the above conditions. This means that the suggestion by $\mathrm{BB}$, that the initial state $B_{+}$be taken to be a $\mathrm{CP}$ eigenstate, is unrealizable in practice.

In order to convince oneself of this fact, one may consider a specific set-up for an experiment of the kind suggested by BB. Suppose that one wanted the initial state to be the $\mathrm{CP}$-odd eigenstate $B_{-}$. At a $B$-factory one uses the decay of the resonance $\Upsilon(4 S)$ to produce a $B_{d} \bar{B}_{d}$ pair in an antisymmetric state; if at a certain instant the meson in the left side of the detector is observed to decay into a $\mathrm{CP}$-even state, we may presume that the meson in the right side of the detector is, at that instant, $B_{-}$.

One must however be careful and study in detail the decay into the specific $\mathrm{CP}$-even state that one uses as a tag. That CP-even state may be, for instance, $\pi^{+} \pi^{-}$. Now, the linear combination of $\left|B_{d}\right\rangle$ and $\left|\bar{B}_{d}\right\rangle$ which decays into $\pi^{+} \pi^{-}$ is

$$
\left|B_{\mathrm{yes}}\right\rangle=\left\langle\pi^{+} \pi^{-}|T| B_{d}\right\rangle^{*}\left|B_{d}\right\rangle+\left\langle\pi^{+} \pi^{-}|T| \bar{B}_{d}\right\rangle^{*}\left|\bar{B}_{d}\right\rangle
$$

indeed, the orthogonal linear combination,

$$
\left|B_{\mathrm{no}}\right\rangle=\left\langle\pi^{+} \pi^{-}|T| \bar{B}_{d}\right\rangle\left|B_{d}\right\rangle-\left\langle\pi^{+} \pi^{-}|T| B_{d}\right\rangle\left|\bar{B}_{d}\right\rangle
$$

clearly cannot decay into $\pi^{+} \pi^{-}$. Thus, if at a certain instant we observed $\pi^{+} \pi^{-}$ in the left side of the detector, we would know the meson in the right side of the detector to be, at that instant, not $B_{-}$as we might presume, but rather $B_{\text {no }}$. This means that

$$
e^{i \zeta}=-\frac{\left\langle\pi^{+} \pi^{-}|T| B_{d}\right\rangle}{\left\langle\pi^{+} \pi^{-}|T| \bar{B}_{d}\right\rangle} .
$$


Assuming the decays to be given by the standard-model tree-level diagrams, we would have

$$
\begin{aligned}
e^{i \zeta} & =-\frac{V_{u b}^{*} V_{u d}\left\langle\pi^{+} \pi^{-}\left|\left[\bar{b} \gamma^{\mu}\left(1-\gamma_{5}\right) u\right]\left[\bar{u} \gamma_{\mu}\left(1-\gamma_{5}\right) d\right]\right| B_{d}\right\rangle}{V_{u b} V_{u d}^{*}\left\langle\pi^{+} \pi^{-}\left|\left[\bar{u} \gamma_{\mu}\left(1-\gamma_{5}\right) b\right]\left[\bar{d} \gamma^{\mu}\left(1-\gamma_{5}\right) u\right]\right| \bar{B}_{d}\right\rangle} \\
& =-\frac{V_{u b}^{*} V_{u d}}{V_{u b} V_{u d}^{*}} e^{i\left(\xi+\xi_{d}-\xi_{b}\right)}
\end{aligned}
$$

because the CP-parity of $\pi^{+} \pi^{-}$is +1 . We would thus obtain

$$
\theta=\zeta-\arg \frac{q}{p}=2 \arg \left(V_{u d} V_{t b} V_{u b}^{*} V_{t d}^{*}\right)(\bmod \pi) .
$$

As expected, $\theta$ is independent of the CP-transformation phases and is rephasinginvariant.

\section{Conclusions}

I conclude that BB's claimed discoveries are spurious. Instead of talking loosely about using CP-eigenstate initial states, it is essential to take into account the exact physical mechanism that one uses to tag the initial state. There is no new form of indirect $\mathrm{CP}$ violation, contrary to what $\mathrm{BB}$ have claimed$\operatorname{Im} \epsilon$ is a CP-violating parameter in the relationship between mixing and the decay amplitudes; the latter originate in the tagging of the initial state. The construction itself of the parameter $\epsilon$ is artificial and devoid of any physical meaning.

\section{References}

[1] M. C. Bañuls and J. Bernabéu, Phys. Lett. B 423 (1998) 151.

[2] F. Bartelt et al. (CLEO Collaboration), Phys. Rev. Lett. 71 (1993) 1680.

[3] The technique used in this and the following sections has been developed in collaboration with Gustavo C. Branco and João P. Silva (to appear). 